\title{
Digital image processing of optical density wave propagation in Dictyostelium discoideum and analysis of the effects of caffeine and
} ammonia

\author{
FLORIAN SIEGERT and CORNELIS WEIJER \\ Zoologisches Institut, Ladwig Maximullians Universität München, Luisenstraße 14, D-8000 München 2, Federal Republic of Germany
}

\begin{abstract}
Summary
Waves of chemotactic movement during the early phase of aggregation in Dictyostelium discoideum were analyzed by digital image processing in a manner that immediately shows the following parameters: wave propagation velocity, period length, wave amplitude und wave shape. We have characterized the aggregation of $A X-2$ and the streamer $F$ mutant NP 377 in terms of these parameters and investigated the influence of caffeine and ammonia. It was found that during normal aggregation oscillation frequency increases while at the same time wave propagation velocity decreases. Caffeine, a known inhibitor of cyclic AMP relay, reduces oscillation frequency and wave propagation velocity in a dose-dependent manner but most notably leads to the appearance of bimodal (harmonic) oscillations. These bimodal waves are also found in streamer $F$ mutants without caffeine during early aggregation.
\end{abstract}

The effect of caffeine is interpreted as an increase in the average chemotactic deadaptation time due to elevated cyclic GMP levels after a cyclic AMP stimulus. This increased deadaptation time results in some cells responding to every chemotactic signal, while others respond only to every second signal, leading to mixed population behavior and hence biphasic optical density waves. Ammonia has no significant influence on oscillation frequency and wave propagation velocity but shows a clear increase in the amplitude of the optical density waves. This may indicate a more vigorous chemotactic response by individual cells or a better synchronization of the responding cell populations due to shortened chemotactic deadaptation times.

Key words: chemotaxis, Dictyostelium, digital image processing, caffeine, ammonia.

\section{Introduction}

Amoebae of Dictyostelium discoideum multiply as single cells. Their developmental cycle is initiated when the food source is exhausted. Upon starvation the amoebae aggregate into clusters of about $10^{5}$ cells, which then form a fruiting body and differentiate into stalk and spore cells. The cells are attracted chemotactically by periodic cyclic AMP signals emitted from the aggregation center (Roos et al. 1975; Tomchik \& Devreotes, 1981). High-magnification time-lapse films reveal that individual cells move toward the center in a periodic fashion. Since all cells at a given distance from the center move simultaneously, this leads to waves of inward cell movement (Alcantara \& Monk, 1974; Gross et al. 1976).

When cyclic AMP binds to the cyclic AMP surface receptors of a cell (Malchow \& Gerisch, 1973; Van Haastert \& De Wit, 1984), the cell responds in two ways: first, it relays the signal by synthesizing and releasing additional cyclic AMP; and second, it moves chemotactically in the direction of the signal. The relay response is initiated by the transient activation of adenylate cyclase (Dinauer et al. 1980a; Theibert \& Devreotes, 1986); extracellular cyclic AMP is destroyed by membranebound and secreted phosphodiesterases. The relay response is followed by a refractory period during which the cells are insensitive (adapted) to cyclic AMP. This adaptation process is responsible for the unidirectional outward relay of the signal (Dinauer et al. 1980b).

Besides the relay response, cyclic AMP induces chemotactic cell movement. Cells move in the direction of increasing cyclic AMP concentration, as a result of waves coming from the aggregation center; they stop moving when the wave has gone past and the cyclic AMP concentration starts falling again. This behavior demonstrates the existence of an adaptation process that terminates the chemotactic response (Futrelle et al. 1982; Van Haastert, 1983; Wurster \& Butz, 1983).

After cyclic AMP stimulation of aggregation-competent cells in suspension there is an immediate increase in cyclic GMP (peak after 10s) and a slower rise in cyclic AMP (peak after $1 \mathrm{~min}$ ) (Wurster et al. 1977; Mato, 
1978; Kesbeke et al. 1986; Newell et al. 1988). Evidence for the involvement of cyclic GMP in chemotaxis comes from the study of streamer F mutants (Ross \& Newell, 1981). Streamers are mutants that form abnormally long aggregation streams and in dark-field optics show wide light bands interspersed with narrow dark bands. Since the light bands are thought to reflect moving cells (see below), it has been suggested that the chemotactic movement response in streamer $\mathrm{F}$ mutants is extremely prolonged (500 s compared to $100 \mathrm{~s}$ in wild-type cells) (Ross \& Newell, 1981). Cyclic AMP-induced cyclic GMP accumulation is enhanced in streamer $F$ mutants as a result of a defect in the structural gene for cyclic GMPspecific phosphodiesterase (Van Haastert et al. 1982; Coukell \& Cameron, 1985). From this observation it was concluded that cyclic GMP is involved in the chemotactic response. The precise role of cyclic GMP is not yet clear but it may be involved in the local polymerization of myosin in the trailing edge of the cell (Liu \& Newell, 1988; Newell et al. 1988).

The pulsatile nature of the cyclic AMP signal can be visualized using low-magnification, low-angle, dark-field optics (Alcantara \& Monk, 1974). Aggregating amoebae on non-nutrient agar show propagating waves of light and dark bands that form concentric rings and spirals. The light bands are made of elongated inward-moving cells, while the amoebae in the dark bands are stationary (Alcantara \& Monk, 1974). The frequency of the waves increases during development either continuously or discontinuously at the time of stream formation (Durston, 1974; Gross et al. 1977). The speed of successive waves decreases but that of an individual wave is constant (Gross et al. 1976).

The relay of the cyclic AMP signal is visible as an outward-moving spiral wave. It has been shown by isotope dilution-fluorography (Tomchik \& Devreotes, 1981; Devreotes et al. 1983) that the outward-moving light bands are identical with the highest cyclic AMP concentration in the aggregation territory.

We have used digital image processing to investigate the temporal and spatial dynamics of this complex twodimensional pattern. Several parameters of aggregation can be directly analyzed: the frequency of the cyclic AMP signal, the velocity of outward-moving waves, the amplitude and the shape of the waves (along the time axis).

We confirmed that during aggregation the frequency of cyclic AMP waves increases while the outward-moving velocity of successive waves decreases. The propagation velocity of a single wave was constant over large distances (up to $20 \mathrm{~mm}$ ). Caffeine, which is a highly specific inhibitor of cyclic AMP relay (Brenner \& Thoms, 1983), caused a change in chemotactic adaptation, which led to bimodal optical density waves. Such bimodal waves were also observed in streamer $F$ mutants and in our earlier work with another streamer mutant HG220 (Gottmann \& Weijer, 1986). Ammonia, which inhibits cyclic AMP relay (Williams et al. 1984; Feit \& Sollitto, 1987) and affects cell movement (Bonner et al. 1986, 1988), had no effect on oscillation frequency, wave propagation speed or wave shape but caused a definite increase in the amplitude of the optical density oscillations.

\section{Materials and methods}

\section{Strains and growth conditions}

Cultures were grown from frozen spores (stored at $-20^{\circ} \mathrm{C}$ ) of strain AX-2. Every three weeks new cultures were started in axenic medium according to standard procedures (Sussmann, 1987 ) at $22^{\circ} \mathrm{C}$. Doubling time was $8 \mathrm{~h}$. The streamer $\mathrm{F}$ mutant NP377 was grown on bacteria (Klebstella aerngenese) on plates or in bacterial suspension, according to standard procedures (Sussmann, 1987).

\section{Preparation of wave plates}

For aggregation experiments cells were harvested at a density of $3 \times 10^{6}$ to $6 \times 10^{6}$ cells ml $^{-1}$. Harvesting cells at lower densities resulted in very poor optical density waves. To initiate development cells were washed by two centrifugations ( $300 \mathrm{~g}$ for $2 \mathrm{~min}$ ) in $20 \mathrm{~mm}-\mathrm{KK} 2$ buffer, $\mathrm{pH} 6 \cdot 8$, (KK2 buffer is potassium phosphate) and resuspended in the same buffer at a density of $5 \times 10^{6}$ cells $\mathrm{ml}^{-1}$. For ammonia experiments the cells were washed and resuspended in $20 \mathrm{~mm}-\mathrm{KK} 2$ buffer, $\mathrm{pH} 7 \cdot 5$. Immediately after centrifugation $5 \mathrm{ml}$ of cell suspension was poured on plates containing $5 \mathrm{ml} \mathrm{KK2}$ buffer-agar $(0.8 \%$ Bacto agar in $20 \mathrm{~mm}-\mathrm{KK} 2$, $\mathrm{pH} \mathrm{6.8;} \mathrm{pH} 7.5$ for ammonia experiments). Caffeine and ammonium chloride were added, when the agar had cooled down to $50^{\circ} \mathrm{C}$. The cells were dispersed uniformly over the agar surface by gentle shaking. After 5-10 min the cells stuck to the agar and the supernatant was poured off. The Petri dishes were kept at $22^{\circ} \mathrm{C}$ in the dark for $4 \mathrm{~h}$ until aggregation started. The visibility of the dark-field waves was strongly influenced by the residual fluid on the agar surface. Best results were obtaned by drying the Petri dishes in a sterile hood for $10-15 \mathrm{~min}$ after $4 \mathrm{~h}$ of development; waves then appeared almost immediately.

\section{Observation of dark-field waves and digital image processing}

A dark-field system was constructed as described by Gross et al. (1976). Instead of a double plano-convex lens we used a single plano-convex lens of $12 \mathrm{~cm}$ diameter and a focal length of $20 \mathrm{~cm}$. A lamp was placed two focal lengths under the lens. Immediately above the lamp there was an annular diaphragm of $2.5 \mathrm{~cm}$ inner diameter; the video camera used to record the waves was placed two focal lengths above the lens. In this position there was a $1: 1$ projection of the diaphragm on the lens. The Petri dish with cells was placed half a focal length above the lens.

The video camera was equipped with a Tokına Zoom $(35-70 \mathrm{~mm})$ objective and time-lapse films of aggregation were made with a time-lapse video recorder (Gyrr). At the same time the video image was digitized at a rate of 25 images $^{-1}$ $(512 \times 512$ pixels with a resolution of 256 gray values). The image-processing board (FG100, Imaging Technology) was installed in an IBM AT compatible computer (Tandon) and special application programs were written by us in MicroSoft C. All board functions were controlled interactively by our program and the processed video images were displayed on an additional monitor.

\section{Data analysis}

For temporal analysis the gray values inside a window (see Fig. 1) were averaged along the short axis to create a onedimensional representation of optical density waves in the aggregation field; the resulting values were saved on a hard disc. As long as dark-field waves were visible, an image was taken every $20 \mathrm{~s}$, the average values along the $x$-axis were calculated and the resulting array was saved. To analyze the kinetics of 

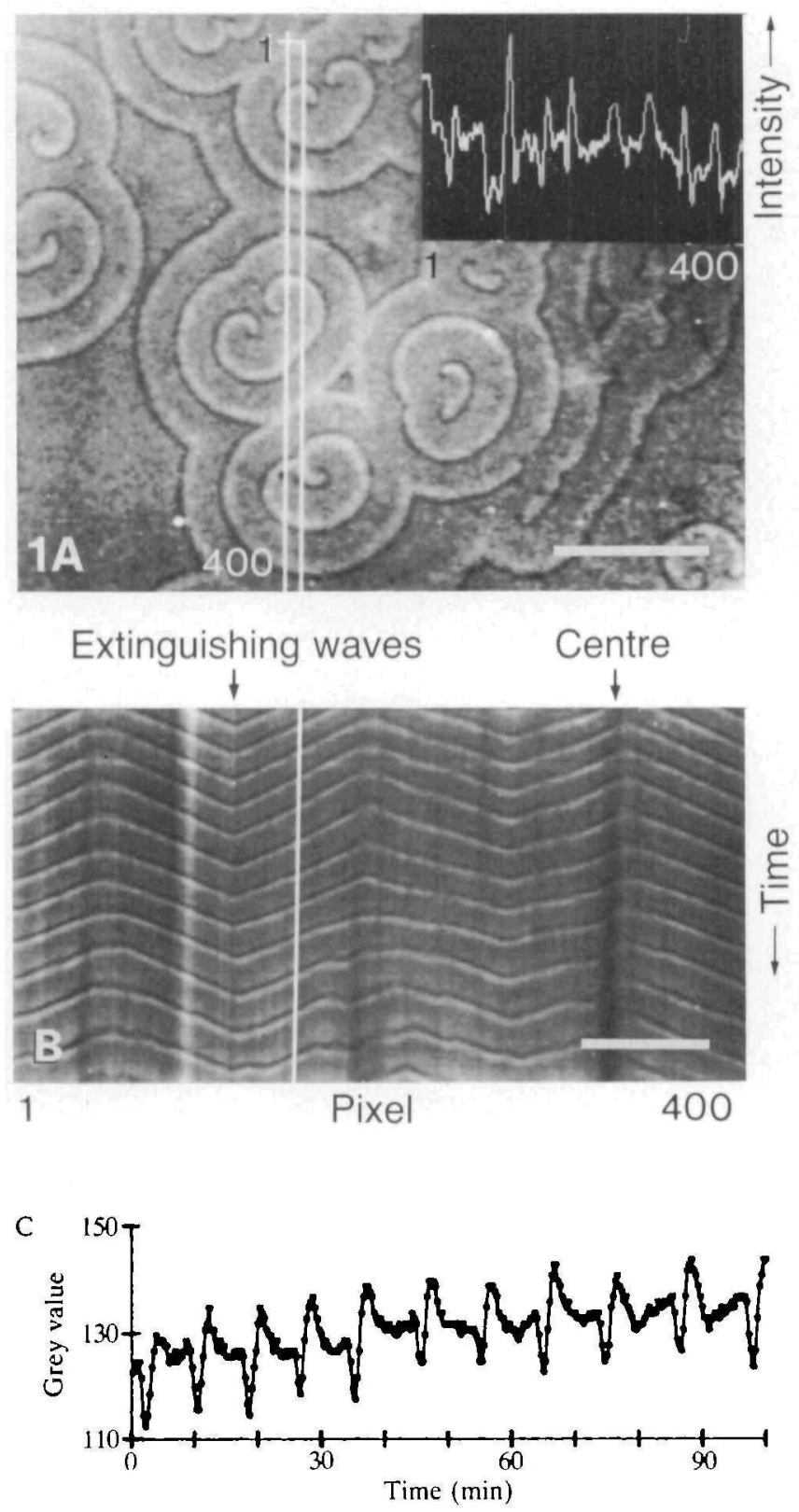

Fig. 1. Digital image processing of optical density waves. A. Aggregation of strain AX-2 on $20 \mathrm{~mm}$-phosphate-buffered agar, $\mathrm{pH} 6 \cdot 8$, containing $3 \mathrm{~mm}$-caffeine. At 20 -s intervals the video signal is digitized as an $512 \times 512$ array of gray values of eight bit resolution. For temporal analysis the data were reduced to a one-dimensional representation: each row of gray values inside the window was averaged along the short axis and the resulting values representing the optical density along the long axis were saved on a hard disc. The graphic inset displays the averaged gray values: left to right in the inset corresponds to top to bottom in the window. Bar,

$10 \mathrm{~mm}$. B. New image generated by representing an optical density scan as a row of pixels across the screen and placing successive scans below each other on the screen. The $y$-axis of the new image represents $100 \mathrm{~min}$ of development from top to bottom. The slope of the bands represents the outwardmoving velocity of the waves and is measured by interactively aligning a line along the bands and calculating the resulting tangent. Bar, $6 \mathrm{~mm}$. C. Gray values along the vertical white line in B plotted against time (1 pixel equals $20 \mathrm{~s}$ ). wave propagation the sequence of stored arrays was read from the disc and displayed sequentially along the $y$-axis. In the resulting time series image (Fig. $1 \mathrm{~B}$ ) the $x$-axis represents the optical density over a section of an aggregation plate at a given moment and the $y$-axis represents time, i.e. the progression of aggregation over this section.

By reading out the frame memory along the $y$-axis of the time series image (Fig. 1B), the changes in wave shape of any given point can be seen and analyzed by further programs. To eliminate differences in lighting the data were normalized according to the following formula: $\left(x_{1}-\Sigma x_{1} / n\right)-1$. As a measure of the amplitude of the oscillations we calculated the standard deviation of all normalized data in a given time series. For power spectrum analysis the data were subjected to filtering through a second-order Butterworth band-pass filter with $60 \mathrm{~s}$ and $3000 \mathrm{~s}$ upper and lower cut-off periods (Gottmann \& Werjer, 1986), before calculating power spectra using the Fast Fourier Transform algorithm.

\section{Results}

\section{Velocity and period of the dark-field waves}

Measurements were started with the appearance of the first waves and continued until the dark-field waves disappeared during the formation of tight cell-cell contacts. Under these conditions $20-35$ waves could be analyzed. Most of the waves were spirals. In some cases we observed concentric rings at the beginning of aggregation, which were normally rapidly converted to spiral structures. A spiral wave is a continuous structure, each part of which propagates outwards from the center. If one examines the pattern along an arbitrarily chosen line through the center of the spiral, it appears as a succession of discrete waves. In light areas the cells are stretched and reflect light strongly; these cells are moving. In dark areas the cells are flattened and reflect less light; such cells are not moving (Alcantara \& Monk, 1974; authors' unpublished observations). The optical density along the long axis of the window shown in Fig. 1A is given in the inset. Increasing gray values correspond to increasing reflected light. These waves move outwards with time and new waves start at regular intervals at the aggregation center.

Fig. 1B represents dark-field waves during $100 \mathrm{~min}$ of aggregation. The image was constructed by listing successive sets of optical density values below each other. As a wave moves outward in the aggregation field it generates a sloped line as shown in Fig. 1B. Several parameters of aggregation are directly apparent from this time series image. The bright and dark bands in Fig. 1B correspond to the bright and dark bands of the dark-field waves. The vertical distance between two successive bright bands is equal to the period length of one wave. The period length was determined by reading a vertical line of pixels out of the frame memory and measuring the distance between the minima of the gray values (Fig. 1C). The slope of the bands represents the velocity of wave propagation. A flat slope represents high velocity, a steep slope low velocity. From Fig. 1B it is evident that each wave travels with nearly constant velocity. Aggregation centers appear in Fig. $1 \mathrm{~B}$ as peaks from which lines slope downwards on both sides. When two travelling waves collide, they 
extinguish each other. In Fig. 1B this event appears when two downward sloping lines meet.

With digital image processing, two further parameters, previously not measurable, could be analyzed. By reading a vertical line of pixels out of the frame memory (Fig. 1B), it was possible to display the amplitude of the light-scattering response and the shape of the travelling wave at a given point in the aggregation field (Fig. 1C). Amplitude of the light-scattering response is determined by the vigor of cell motion, i.e. the degree of light scattering by individual cells, and by the degree of synchrony among moving cells. The shape of the wave therefore yields information about the variation in chemotactic reaction in a population of cells.

The velocity of successive waves declined from $500 \mu \mathrm{m} \mathrm{min}^{-1}$ at the beginning of aggregation to $260 \mu \mathrm{m} \mathrm{min}^{-1}$ at the end of aggregation (Figs 2D, 3A). At the same time the period decreased from $5.8 \mathrm{~min}$ to $4.5 \mathrm{~min}$ (Figs $2 \mathrm{D}, 3 \mathrm{~B}$ ). Because of the velocity changes, the period was measured close to the aggregation center. After formation of cell-cell contacts the period appears to decrease further, since we have measured periods of $2 \mathrm{~min}$ in cell streams of streamer mutants. These results demonstrate that the propagation velocity decreases during aggregation while at the same time the frequency increases (Fig. 3A,B).

\section{The influence of caffeine on aggregation}

Caffeine ( $3 \mathrm{~mm}$ ) reduces cyclic AMP production to $5 \%$ of the normal level while cyclic GMP synthesis increases (Brenner \& Thoms, 1983; Van Haastert \& De Wit, 1984). Caffeine should therefore influence cyclic AMP relay as well as chemotactic movement.

Effect of caffeine on wave propagation speed and oscillation frequency. Treatment of aggregating cells with 1-3 $\mathrm{mM}$-caffeine enhanced visibility and stability of the dark-field waves compared to controls. Caffeine influenced all measured parameters of aggregation, except the amplitude. Fig. 2 shows a comparison of darkfield waves on agar containing 0,3 and $5 \mathrm{~mm}$-caffeine with the corresponding time series images. Cyclic AMP production is most probably strongly inhibited under these conditions, although we have not measured it directly. This could only be done by isotope dilution fluorography experiments as described by Devreotes (Tomchik \& Devreotes, 1981), which was outside of the scope of this paper. Even $5 \mathrm{~mm}$-caffeine does not block aggregation and the cells are able to form mounds.

Comparison of the images in Fig. 2 indicates that aggregation territories become much larger in the presence of caffeine (same scale on all images). The velocity of individual waves on caffeine remains constant over large distances (up to $20 \mathrm{~mm}$ ) ; however, the wave propagation velocity on caffeine is reduced compared to untreated controls (Fig. 3A). Caffeine also changes the dynamics of velocity decrease during aggregation: velocity decreases in untreated controls in a sigmoid manner, while in the presence of caffeine the decrease in velocity is nearly linear. The sigmoid decrease in velocity seen here in AX-2 contrasts with the linear decrease in velocity seen in NC4 by Gross et al. (1976). The reason for this discrepancy is not clear; it could result from differences between the strains or be due to the higher resolution of our method for determining velocities (which measures propagation of each single wave over large distances). The period between successive waves is longer than in untreated controls and, instead of getting shorter, the period length increases during aggregation (Fig. 3B). We also measured aggregation on $1 \mathrm{~mm}$ and 2 mm-caffeine (data not shown) and found these effects to increase in a dose-dependent manner.

Changes in wave shape. Analysis of the grey values along the time axis shows that there is an obvious difference in wave shape between control cells and caffeine-treated cells: untreated control cells exhibit distinct light and dark bands of the same width. In the presence of caffeine the light band remains the same while the dark band becomes much broader (Fig. 2A,B,C). In addition a very narrow dark band develops at the leading edge of the light bands (Fig. 2B,C). Fig. 5 shows a comparison of untreated control cells with $5 \mathrm{~mm}$-caffeine-treated cells. The increase in light scattering is in both cases very similar while the decrease takes twice as long with caffeine. Additionally, in later stages of aggregation the waves become bimodal with caffeine.

Analysis of the power spectra of waves in the presence and absence of caffeine revealed a single peak (frequency) for control cells but two harmonic frequencies for caffeine-treated cells (Fig. 4). This means that the main signal, which contains most of the energy, is superimposed on a second signal with twice the frequency and less energy. This result was obtained with all tested caffeine concentrations $(1,2,3,5 \mathrm{~mm})$ in every experiment. Furthermore, it can be seen that increasing concentrations of caffeine slow down overall frequency.

The effect of caffeine on amplitude of the optical density waves. The amplitude of dark-field waves is correlated with the intensity of the light-scattering properties of the cells and possibly reflects the velocity of cell movement. After normalizing the data to eliminate differences in lighting between experiments no difference in amplitude between controls and caffeine plates could be detected (Figs 4, 5).

Effects of caffeine on center morphology and cringe response. Two other effects of caffeine are apparent from wave patterns and time series images. First, the so called cringe response (Futrelle et al. 1982) (visible as a very narrow dark band immediately preceding each light band, see Fig. 2B,C) is intensified by caffeine. This impression could be caused by the brighter average gray level in caffeine-treated cells. Alternatively, caffeine could indeed alter the behavior of the cells and cause an even more rounded shape for stationary cells. Second, aggregation centers on caffeine plates appear to be 'black holes' (see Fig. 2B,C) with no cells inside. Under these conditions cells tend to form rings as aggregation centers (Fig. 6). This effect depends on the caffeine concentration: the more caffeine the bigger the holes.

\section{Analysis of streamer $F$ mutants}

The wave pattern of cells aggregating in the presence of 

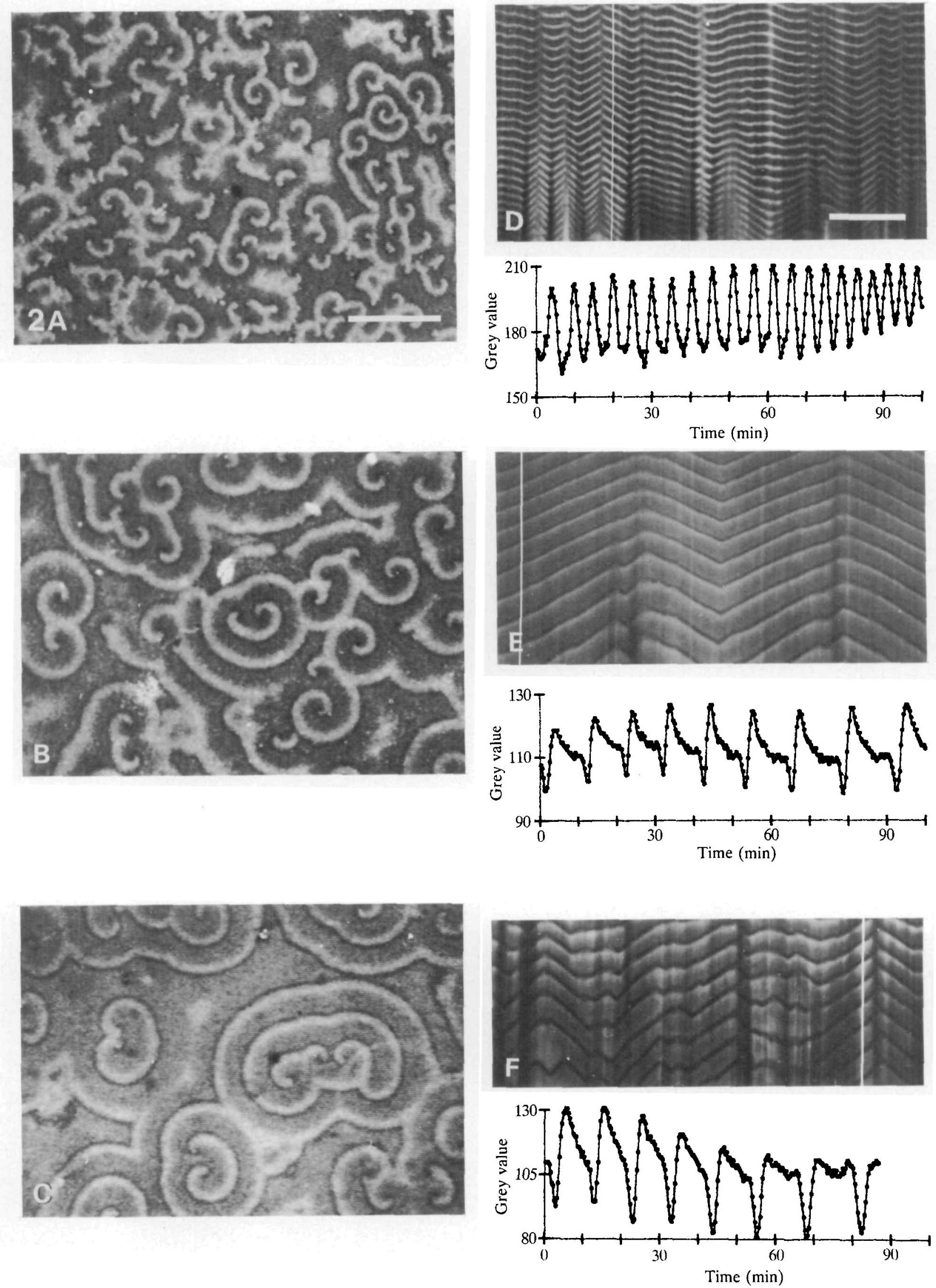

Fig. 2. The influence of caffeine on wave propagation: A-C. Photographs of aggregation on buffered agar containing $0,3 \mathrm{~mm}$ and $5 \mathrm{~mm}$-caffeine. Bar, $10 \mathrm{~mm}$. D-F. Images showing wave propagation and wave form on $0,3 \mathrm{~mm}$ and $5 \mathrm{~mm}$-caffeine. The graph below each video image displays the gray values along the white line (0, black; 255, bright white). Bar, $6 \mathrm{~mm}$. 

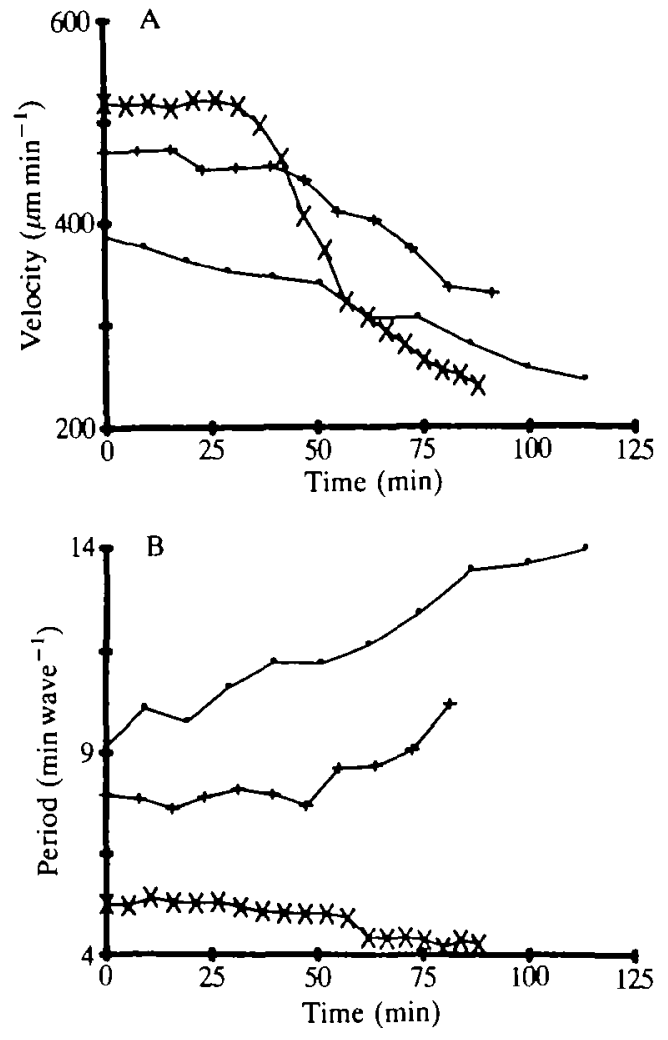

Fig. 3. Analysis of wave propagation speed (A) and period length (B) with $0 \mathrm{~mm}(X), 3 \mathrm{~mm}(+)$, and $5 \mathrm{~mm}(O)$ caffeine. The data represent the average values of $8-12$ centers in $4-6$ experiments for each concentration. The standard deviation is less than $10 \%$.

caffeine resembles that of streamer $\mathrm{F}$ mutants (Ross \& Newell, 1981). These mutants also show a pattern of narrow dark bands separated by broad bands (Fig. 7A). In the case of streamer $\mathrm{F}$ mutants the broad bands are uniformly light; in the case of caffeine the broad bands are a $1 / 4$ light and $3 / 4$ dark (Figs $2 B, C, 7 A$ ).

We investigated the streamer F mutant NP 377 with the methods described above. Wave velocity and frequency were similar to values in wild-type cells (not shown). Analysis of the wave shape during early stages of aggregation showed bimodal signals (Fig. 7B). The power spectrum also showed a clearly bimodal signal (Fig. 7C). Since waves were visible in these mutants for longer periods of time, we could observe a spontaneous change to high-frequency oscillations later in aggregation. Coincident with this frequency change the wave shape became symmetric and the wide light bands disappeared (Fig. 8).

\section{The influence of ammonia on aggregation parameters}

Ammonia influenced only two of the measured aggregation parameters: it inhibited aggregation center forma-
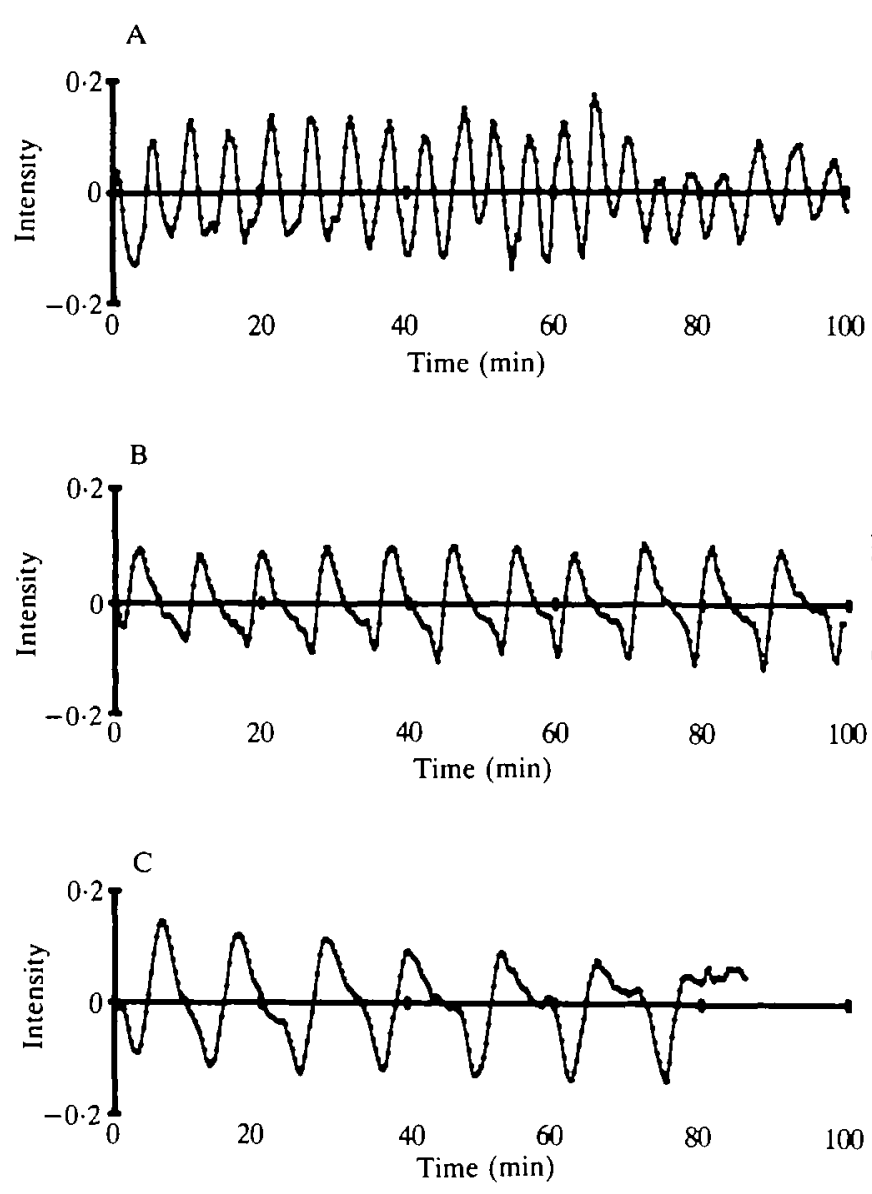
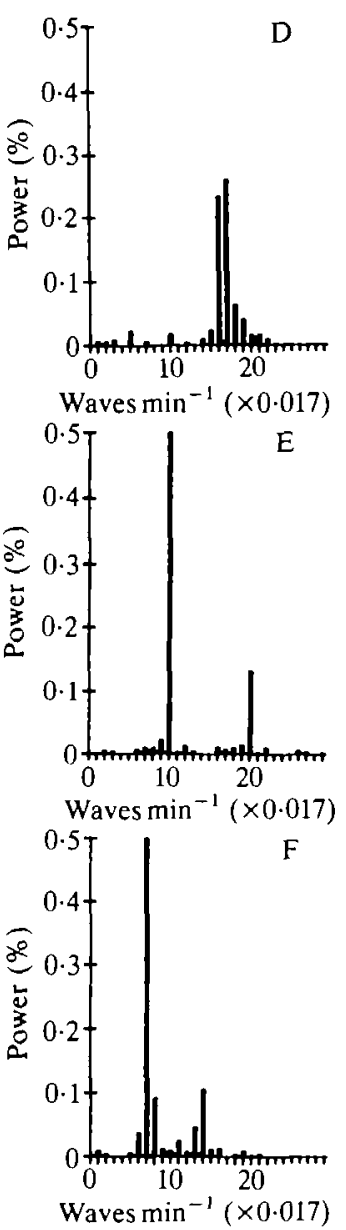

Fig. 4. Analysis of wave form with increasing caffeine concentrations. A-C. Wave forms of experiments with $0,3 \mathrm{~mm}$ and $5 \mathrm{~mm}$ caffeine. D-F. Power spectra of data in A-C. The original data were normalized and filtered as described in Materials and methods and then the power spectra were calculated. 


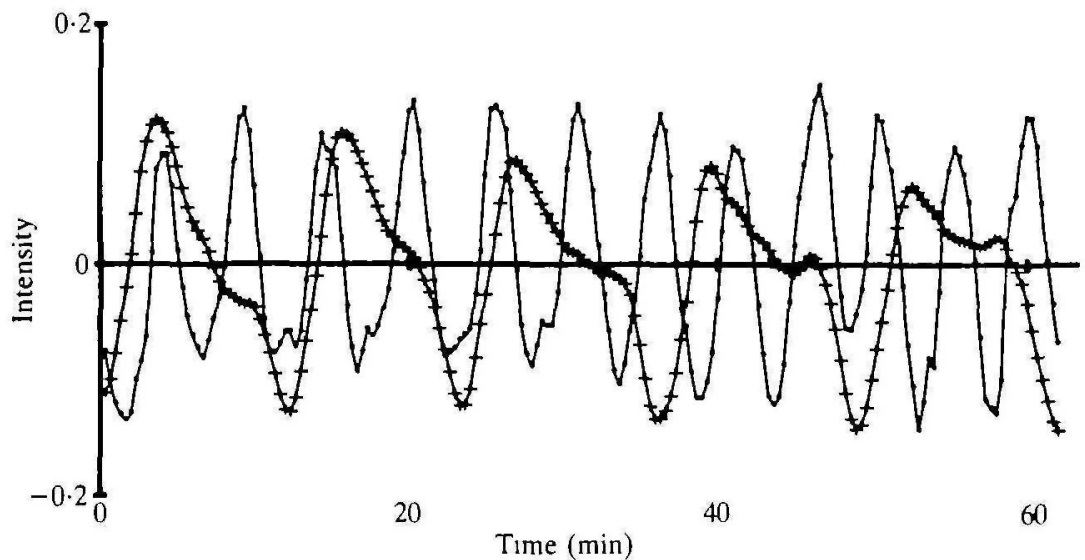

Fig. 5. Comparison of the amplitudes of the dark-field waves without $(O)$ and with $5 \mathrm{~mm}$ caffeine $(+)$. The amplitudes are about the same. The rising phase of the signal is similar in both cases while the falling phase is very different with caffeine, and bimodal towards the end. tion and it increased the amplitude of the optical density oscillations. Fig. 9 shows that oscillation frequency and wave shape were not altered. There was also no measurable effect on wave velocity (data not shown). Ammonia inhibits aggregation center formation (Lonski, 1976) and therefore leads to an enlargement of aggregation territories in a dose-dependent manner, with maximal effect at $2 \mathrm{~mm}$. Fig. 10 shows the effect of ammonia on the amplitude of the optical density oscillations. The maxi- mal effect is seen at $2 \mathrm{~mm}$, the lowest concentration tested.

\section{Discussion}

The dynamics of aggregation and the dispersion relation We have observed an interesting correlation between outward-moving velocity and period of the dark-field waves during aggregation. The frequency increases while
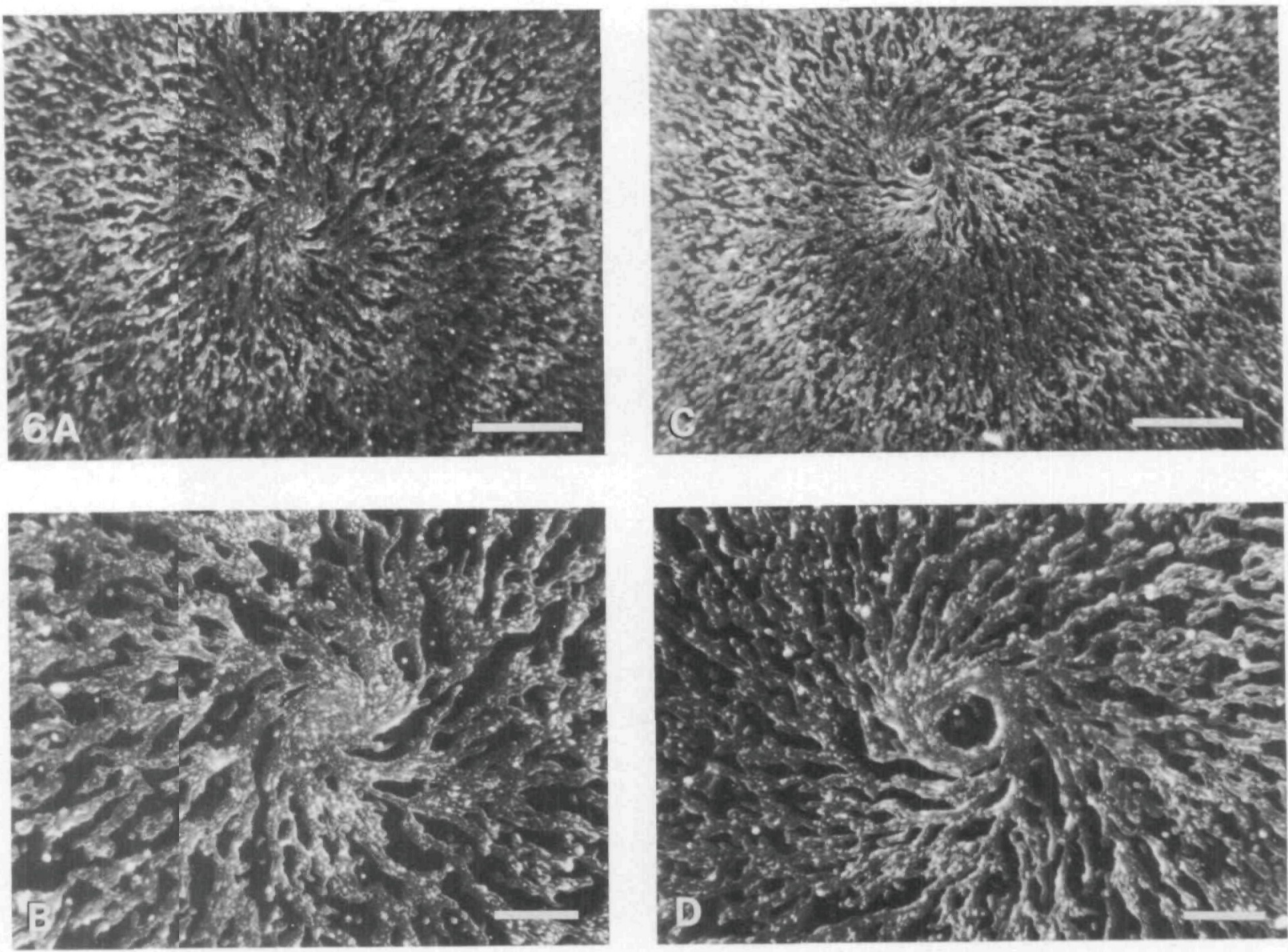

Fig. 6. Effect of caffeine on aggregation centers. A,B. No caffeine. The optical density wave is visible as a light spiral emanatıng from the center. C, D. 2 mm-caffeine. The core of the spiral wave is free of cells. The core diameter depends on the caffeine concentration. Bars: $0.4 \mathrm{~mm}(\mathrm{~A}, \mathrm{C}) ; 0.1 \mathrm{~mm}(\mathrm{~B}, \mathrm{D})$. 

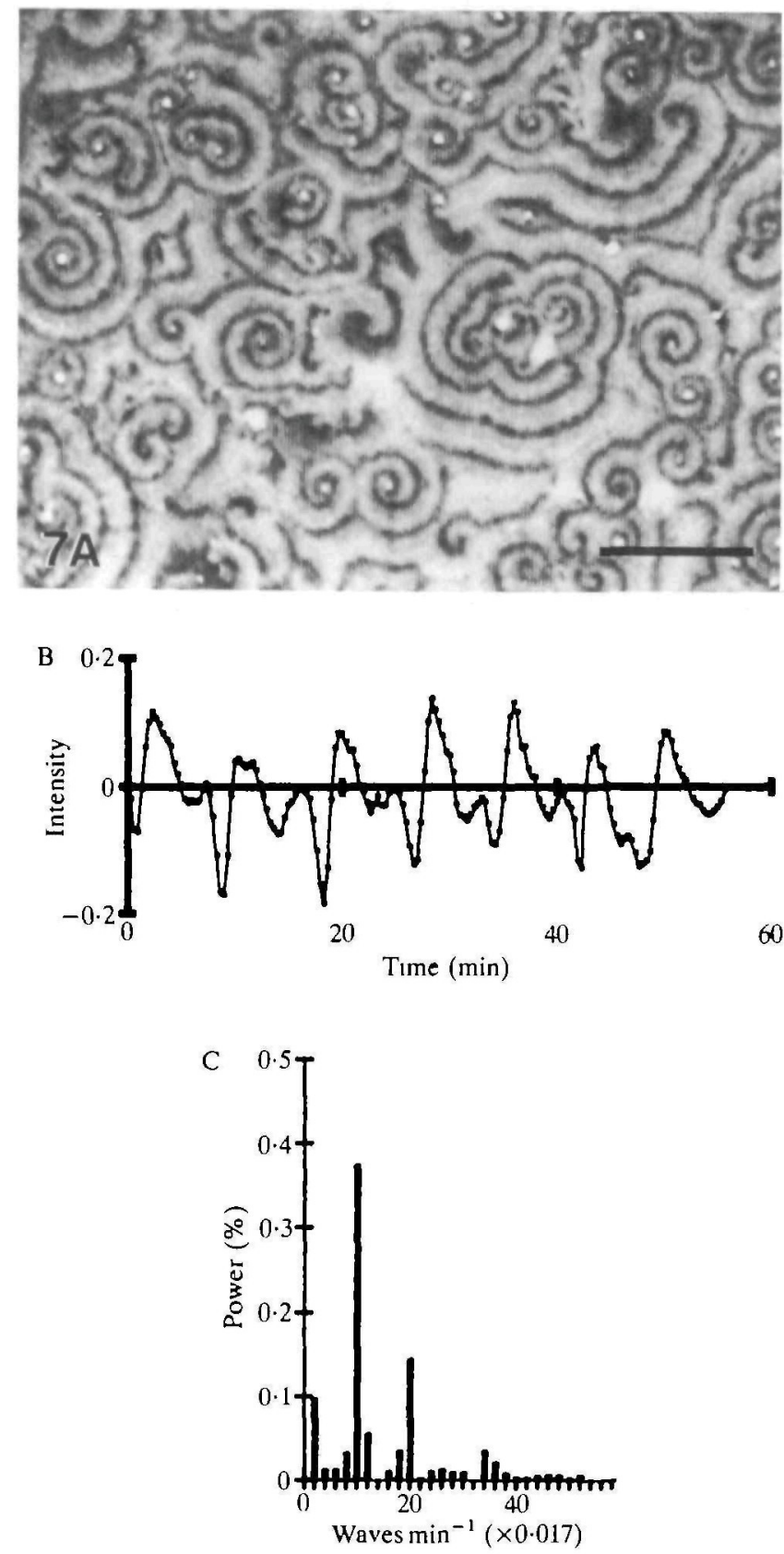

Fig. 7. Wave propagation in a streamer $\mathrm{F}$ mutant (NP377) during early aggregation. A. Photograph of dark-field waves showing broad light bands. Bar, $10 \mathrm{~mm}$. B. Typical wave form showing bimodal signals. C. Power spectrum of data in $\mathrm{B}$.

at the same time the velocity decreases. A similar correlation was reported by Gross et al. (1976, 1977). This phenomenon is also observed in excitable chemical systems like the Belousouv-Zabotinsky reaction (Dockery et al. 1988). The relationship between oscillation frequency and wave propagation velocity is called the dispersion relation. The exact form of the dispersion relation is characteristic of a particular reaction and determined by the underlying chemical kinetics (Dockery et al. 1988). Dispersion relations for the dark-field waves are shown in Fig. 11. As can be seen, AX-2 cells and streamer F mutant NP377 show significantly different dispersion relations. Treatment of AX-2 cells with caffeine leads to a third type of dispersion relation: the velocity decreases while the period length increases. Thus, in streamers and in caffeine-treated cells different reactions must be affected. The results put constraints on possible models of the underlying reactions and may be used to discriminate between various possible models.

Caffeine causes a decrease in frequency and propagation velocity of the light-scattering response. Aggregation territories get bigger and the aggregation centers tend to form rings under the influence of caffeine (Fig. 6). The diameter of the rings becomes larger with increasing caffeine concentration. This supports a suggestion by Durston (1973) that the spiral core is formed by the continuous circulation of one excitation wave around a refractory center. Since caffeine lengthens the period, one would indeed expect larger spiral cores.

\section{Relationship between signal strength and amplitude of} the optical density waves

Caffeine $(5 \mathrm{~mm})$ reduces cyclic AMP production to less than $2 \%$ of the control levels (unpublished observations); nevertheless, the amoebae are still able to aggregate efficiently. Thus, $D$. discoideum cells can aggregate under conditions where there is at least a fiftyfold difference in signal strength, which may explain why the cells can aggregate over a wide range of cell densities.

It was surprising to observe that the amplitude of optical density waves does not correlate with cyclic AMP signal strength: cells aggregating on $5 \mathrm{~mm}$-caffeine exhibited optical density waves with the same amplitude as cells aggregating in the absence of caffeine. If the amplitude of the optical density waves reflects chemotactic vigor, then this indicates that chemotaxis must somehow be proportional to a relative change in signal strength. Alternatively, it is possible that, even under conditions where the signal amplitude is reduced fiftyfold, most of the receptors remain occupied due to their high affinity (Van Haastert \& De Wit, 1984).

The effect of caffeine on wave shape

Digital image processing has permitted for the first time analysis of the shape of dark-field waves, i.e. the increase and decrease in light-scattering intensity as a reaction to the cyclic AMP signal. The wave shape is symmetric in wild-type cells. Under the influence of caffeine it becomes strongly asymmetric and finally bimodal. Analysis of the power spectra has shown that the signals are composed of two harmonic oscillations and that with increasing caffeine concentration the period length increases.

Changes in the shape of the optical density waves can in principle result from changes in the shape of the cyclic AMP signal or alternatively from changes in the chemotactic response. In either case such changes result from changes in deadaptation time of the response (Gottmann $\&$ Weijer, 1986). If the deadaptation time of the relay response is varied, the shape of the propagated cyclic AMP signal changes and hence the shape of optical 

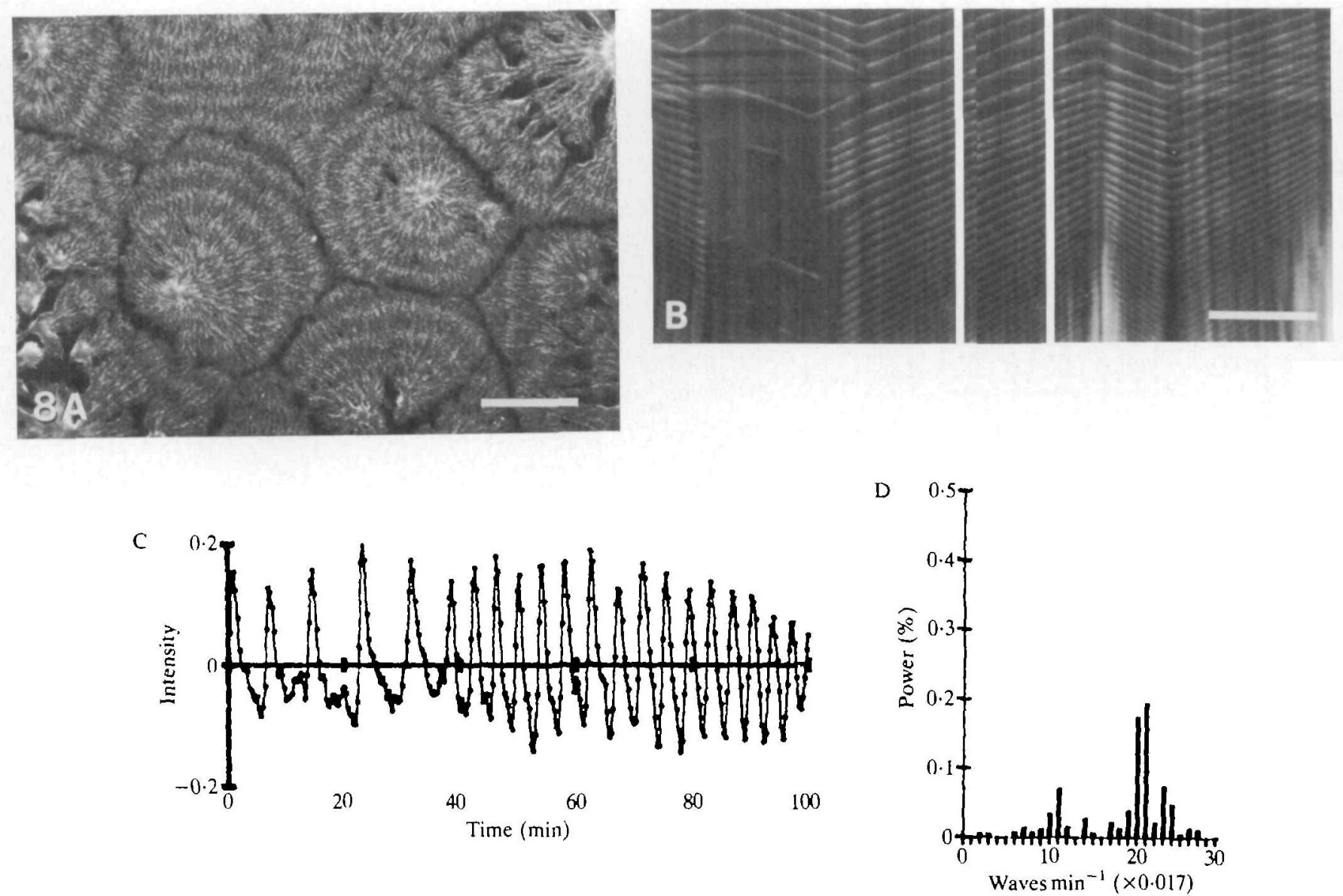

Fig. 8. Wave propagation in a streamer F mutant (NP377) during later aggregation. A. Photograph of dark-field waves. Bar, $2 \mathrm{~mm}$. B. Resulting image from time series. Bar, $6 \mathrm{~mm}$. C. Wave shape along the white line in B, showing a typical frequency doubling. D. Power spectrum.

density waves as well. This does not appear to be a likely explanation for the observations presented here, since caffeine has been shown to have no affect on adaptation and deadaptation times of the relay response (Theibert \& Devreotes, 1983). Hence it appears more likely to us that the deadaptation time of the chemotactic response is affected by caffeine treatment.

The asymmetric shape of dark-field waves in the presence of caffeine appears to be due to the heterogeneous chemotactic response of the cells. We suppose that there is a continuous (normal) distribution of the deadaptation times in the population: some cells deadapt quickly while other cells deadapt more slowly. In the absence of caffeine all cells have deadaptation times that permit them to respond to every cyclic AMP signal. In the presence of caffeine we imagine that the distribution of deadaptation times is shifted towards longer times. Cells that have a deadaptation time longer than the period length of the cyclic AMP signal coming from the aggregation center will not be able to respond to every signal. Cells with shorter deadaptation times will respond to every cyclic AMP signal. The population consists of some

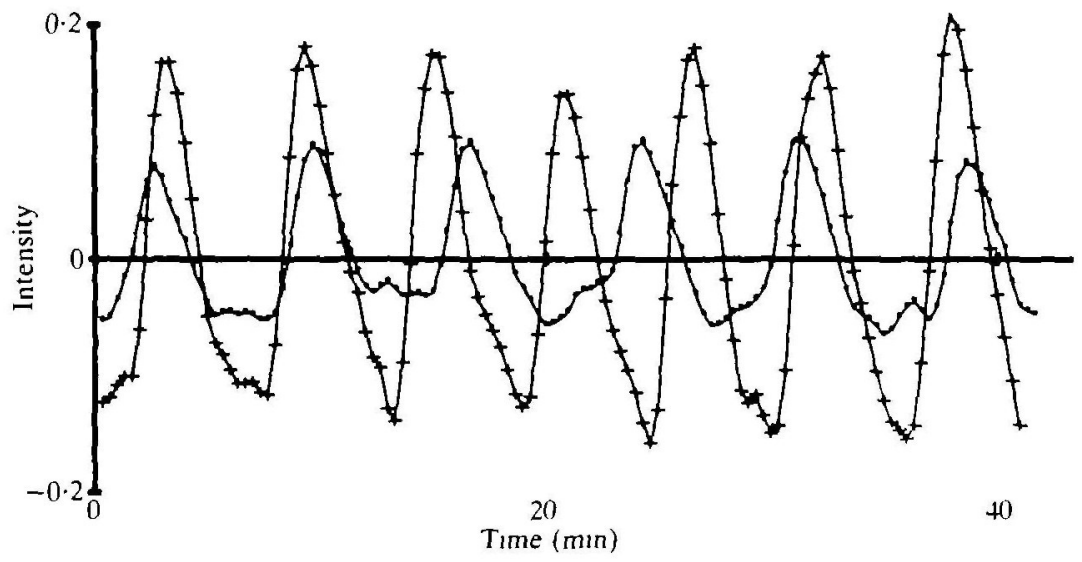

Fig. 9. The effect of $\mathrm{NH}_{3}$ on wave shape. Comparison of frequency, amplitude and wave shape without $(1)$ and with $2 \mathrm{~mm}$ $\mathrm{NH}_{4} \mathrm{Cl}(+)$. 


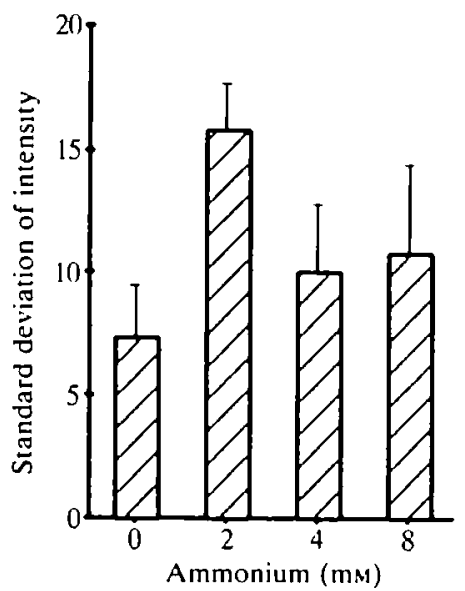

Fig. 10. Comparison of the wave amplitudes in cells aggregating on $0 \mathrm{mM}(n=7), 2 \mathrm{mM}(n=11), 4 \mathrm{mM}(n=13)$ and $8 \mathrm{~mm}-(n=11) \mathrm{NH}_{4} \mathrm{Cl}$ ( $n=$ number of centers measured). The amplitude is quantified by calculating the standard deviation of all data points in one time series. The mean of the standard deviation of several centers is shown for each ammonium concentration and the error bar represents the standard deviation of these measurements. The difference between 0 and $2 \mathrm{~mm}$-ammonium is highly significant $(P<0.001)$. Plates were prepared as described in Materials and methods.

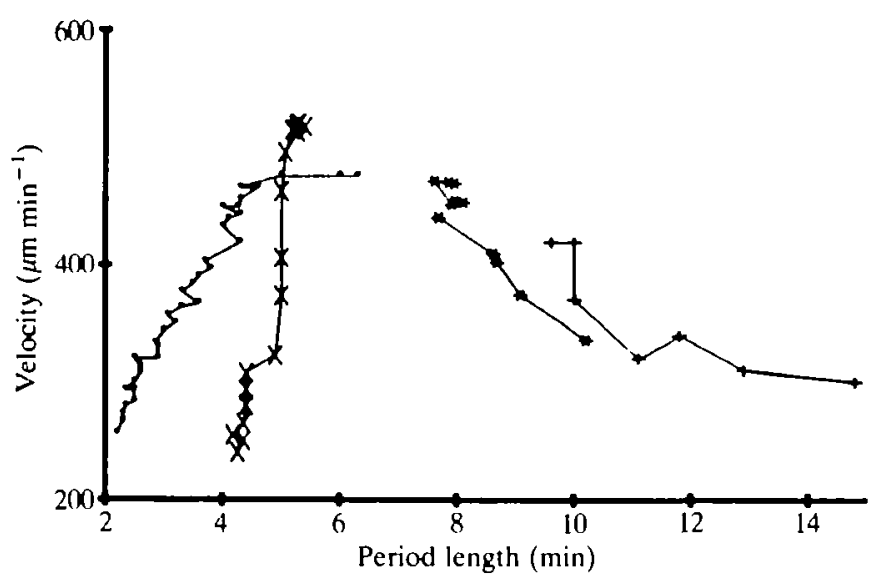

Fig. 11. Relationship between wave propagation velocity and period length. The dispersion relation is shown for AX-2. $0 \mathrm{~mm}(\times), 3 \mathrm{~mm}(*), 5 \mathrm{~mm}(+)$ caffeine and streamer mutant NP377 (O). Developmental time is in the direction of decreasing velocity.

cells that respond to every signal and other cells that respond to every second signal. Thus the dark-field waves exhibit two signals that are harmonic.

This hypothesis also explains why the wave shape during aggregation in the presence of caffeine slowly becomes more biphasic (Figs 4B,C, 5). The results in Figs 3 and 4 show that the period length of the dark-field wave increases during the course of aggregation in the presence of caffeine. Thus, more cells in the population are able to respond to every cyclic AMP signal under the same distribution of deadaptation times.

\section{Measurements on streamer $F$ mutants}

Since the aggregation pattern of wild-type strain AX-2 on caffeine resembles that of streamer $F$ mutants without caffeine, we investigated dark-field waves in the streamer F mutant NP377 for comparison. During early aggregation this mutant clearly shows bimodal oscillations (Fig. 7B), which on power-spectrum analysis also appear to be harmonic oscillations. Later in development the broad light bands disappear (Fig. 8) and the optical density waves closely resemble those of wild-type cells. This is a result of the second peak of the bimodal response developing into a full chemotactic response. This behavior suggests that the deadaptation time in streamer $\mathrm{F}$ cells becomes shorter later in development in such a way that all cells are able to respond to every cyclic AMP signal.

It has been shown that streamer $F$ mutants early in development have a very much reduced cyclic GMP phosphodiesterase activity (2\% of wild-type; Ross \& Newell, 1981; Van Haastert et al. 1982), which leads to a two- to fourfold overproduction of cyclic GMP upon stimulation by cyclic AMP. Results of experiments by Coukell et al. (1984) show that this defect disappears later in development, perhaps because cyclic GMP phosphodiesterase is a developmentally regulated enzyme whose activity increases five- to tenfold during contact formation. Thus normalization of the cyclic GMP phosphodiesterase levels in streamer $\mathrm{F}$ mutants is correlated with normalization of the dark-field waves later in development.

\section{Deadaptation times and cyclic GMP levels}

The size of the cyclic GMP response following cyclic AMP stimulation has been shown to increase 1.5- to fourfold in cells treated with caffeine (Brenner $\&$ Thoms, 1983; Van Haastert \& De Wit, 1984). Streamer F mutants have also been shown to have elevated levels of cyclic GMP, following cyclic AMP stimulation. As discussed above, both caffeine-treated cells and streamer $F$ mutants exhibit bimodal dark-field waves that are quite similar to each other (compare Figs 4B,C and 7B). These bimodal waves appear to result from longer deadaptation times in the presence of caffeine or in streamer $F$ mutants compared to untreated wild-type cells. Thus it appears likely to us that the chemotactic deadaptation time is regulated by the cyclic GMP level in cells.

\section{The effect of ammonia on wave shape}

Ammonia leads most prominently to an increase in the amplitude of the observed optical density waves. This result is most easily explained by an increase in synchrony in the responding population of cells caused by a shortening of the average chemotactic deadaptation time. Bonner et al. (1986) have shown that ammonia stimulates the rate of movement of cells in aggregation streams. Therefore, the increase in amplitude could result from a more vigorous chemotactic response, e.g. more elongated cells. Analysis of movement of single cells under these conditions will allow one to distinguish between these possibilities. 
We thank Peter C. Newell for the generous gift of the mutant NP377. We thank Drs Charles David and Jeff Segel for critical reading of the manuscript. This work was supported by grant no. WE-1127 from the Deutsche Forschungsgemeinschaft.

\section{References}

AlCantara, F. \& MONK, M. (1974). Signal propagation during aggregation in the slime mould Dictyostelium discoideum. $\mathcal{F}$. gen. Microbiol. 85, 321-334.

Bonner, J. T., Chiang, J. L. \& Suthers, H. B. (1988). The possible role of ammonia in phototaxis of migrating slugs of Dictyostelium discoideum. Proc. natn. Acad. Sci. U.S.A. 85, $3885-3887$

Bonner, J. T., Suthers, H. B. \& Odell, G. M. (1986). Ammonia orients cell masses and speeds up aggregating cells of slime moulds. Nature, Lond. 232, 630-632.

Brenner, M. \& Thoms, S. (1983). Caffeine blocks activation of cyclic AMP synthesis in Dictyostelium discoideum. Devl Biol. 101 136-146.

Coukell, M. B. \& Cameron, A. M. (1985). Genetic locus (stmF) associated with cyclic GMP phosphodiesterase activity in Dictyostelum discordeum maps in linkage group II. J. Bact. 162, $427-429$

Coukell, M. B., Cameron, A. M., Pitre, C. M. \& Dee, J. D. (1984). Developmental regulation and properties of the cGMP specific phosphodiesterase in Dictyostelium discoideum. Devl Biol. $103,246-257$.

Dinauer, M., Mackay, S. \& Devreotes, P. (1980a). Cyclic 3': 5'AMP relay in Dictyostelium discoideum. III. The relationship of cAMP synthesis and secretion during the cAMP signal response. $\mathcal{F}$. Cell Biol. 86, 537-544.

Dinauer, M., Steck, T. \& Devreotes, P. (1980b). Cyclic $3^{\prime}: 5^{\prime}$ AMP relay in Dictyostelium discoideum. V. Adaptation of the cAMP signaling response during cAMP stimulation. 7 . Cell Biol. 86, 554-561.

Devreotes, P. N., Potel, M. \& Mackay, S. (1983). Quantitative analysis of cyclic AMP waves mediating aggregation in Dictvostelium discoideum. Devl Biol. 96, 405-415.

Dockery, D., KeENer, J. P. \& Tyson, J. (1988). Dispersion of traveling waves in the Belousov-Zhabotinskyı reaction. Physica $D$ 30, $177-191$.

Durston, A. J. (1973). Dictyostelium discoideum aggregation fields as excitable media. F. theor. Biol. 42, 483-504.

DURSTON, A. J. (1974). Pacemaker activity durıng aggregation in Dictyostelum discoideum. Devl Biol. 37, 225-235.

Fert, I. N. \& SollitTo, R. B. (1987). Ammonia is the gas used for the spacing of fruiting bodies in the cellular slime mould Dictyostelium discoideum. Differentiation 33, 193-196.

Futrelle, R, P., Traut, J. \& McKee, W. G. (1982). Cell behavior in Dictyostelium discoideum preaggregation response to localızed cyclic AMP pulses. 7 . Cell Biol. 92, 807-821.

GotTManN, K. \& WeiJer, C. J. (1986). In situ measurements of external $\mathrm{pH}$ and optical density oscillations in Dictyostelium discondeum aggregates. 7. Cell Biol. 102, 1623-1629.

Gross, J. D., Kay, R., Lax, A., Peacey, M., Town, C. \& Trevan, D. (1977). Cell contact, signaling and gene expression in Dictyostelium discoideun. In Development and Differentiation in the Cellular Slime Moulds (Cappuccinell, P. \& Ashworth, J. M., eds), pp. 135-147. Elsevier/North-Holland Biomedical Press.

Gross, J. D., Peacey, M. J. \& Trevan, D. J. (1976). Signal emission and signal propagation during early aggregation in Dictyostelium discoideum. F. Cell Sci. 22, 645-656.
Kesbeke, F., Van Haastert, P. \& Schaap, P. (1986). Cyclic AMP relay and cyclic AMP-induced cyclic GMP accumulation during development of Dictyostelium discoideum. MS. Micmbiol. Lett. 34, $85-89$.

LIU, G. \& NewELL, P. (1988). Evidence that cyclic GMP regulates myosin interaction with the cytoskeleton during chemotaxis of Dictyostelum. J. Cell Sci. 90, 123-129.

LoNSKI, J. (1976). The effect of ammonia on fruiting body size and microcyst formation in the cellular slime moulds. Devl Biol. 51, $158-165$

Malchow, D. \& Gerisch, G. (1973). Cyclic AMP binding to living cells of Dictyostelium discoideum in the presence of excess cyclic GMP. Biochem. biophys. Res. Commun. 55, 200-204.

MATO, J. M. (1978). ATP increases chemoattractant induced cyclic GMP accumulation in Dictyastelium discoideum. Biochim. biophys. Acta 540, 408-411.

Newell, P., Europe-Finner, G., Small, N. \& LiU, G. (1988). Inositol phosphates, $G$-proteins and ras genes involved in chemotactic signal transduction of Dictyostelium. I. Cell Sci. 89 , $123-127$

Roos, W., Nanjundiah, V., Malchow, D. \& Gerisch, G. (1975). Amplification of cAMP signals in aggregating cells of Dictyostelium discoideum. FEBS Lett. 53, 139-142.

Ross, F. \& NewelL, P. (1981). Streamers: chemotactic mutants of Dictyostelium discoideum with altered cyclic GMP metabolism. $\mathcal{Y}$. gen. Microbiol. 127, 339-350.

SussmanN, M. M. (1987). Cultivation and synchronous morphogenesis of Dictyostelium under controlled experimenta conditions. In Methods in Cell Biology, vol. 28, Dictyostelium discoideum: Molecular Approaches to Cell Biology (ed. Spudich, J. A.), pp. 9-29. Academic Press, NY.

Theibert, A. \& Devreotes, P. (1983). Cyclic 3',5'-AMP relay in Dictyostelium discoideum: Adaptation is independent of activation of adenylate cyclase. 7. Cell Biol. 97, 173-177.

Theibert, A. \& Devreotes, P. (1986). Surface receptor-mediated activation of adenylat cyclase in Dictyostelium. J. biol. Chem. 26, $15121-15124$

'TomchiK, K. \& DeVreotes, P. (1981), Adenosine 3': 5' monophosphate waves in Dictyostelium discoideum: A demonstration by Isotope Dilution-Fluorography. Science 212, 443-446.

VAN HaAstert, P. (1983). Sensory adaptation of Dictyostelium discoideum cells to chemotactic signals. J. Cell Biol. 96, 1559-1565.

VAN HaAsterT, P. \& DE WIT, R. (1984). Demonstration of receptor heterogeneity and affinity modulation by nonequilibrium binding experıments. 7. biol. Chem. 259, 13321-13328.

Van Haastert, P., van Lookeren Campagne, M. M. \& Ross, F. M. (1982). Altered cGMP phosphodiesterase activity in chemotactic mutants of Dictyostelium discoideum. FEBS Lett. 147, $149-152$.

Williams, G. B., Elder, E. M. \& Sussman, M. (1984). Modulation of the cAMP relay in Dictyostelium discoideum by ammonia and other metabolites: possible morphogenetic consequences. Devl Btol 105, 377-388.

WURSTER, B. \& BUTZ, U. (1983). A study on sensing and adaptation in Dictyostelium discoideum: Guanosine $3^{\prime} 5^{\prime} \cdot$ phosphate accumulation and light-scattering responses. F. Cell Biol. 96 $1566-1570$.

Wurster, B., Schubiger, K., Wick, U. \& Gerisch, G. (1977) Cyclic GMP in Dictyostelium. Oscillations and pulses in response to folic acid and cyclic AMP signals. Cell Biol. Int. Rep. 2, 61-69.

(Received 24 January 1989 - Accepted 21 March 1989) 
